\title{
Sense of coherence and health-related quality of life in patients with neurotoxicity after cancer chemotherapy: Assessment from a real-time mobile phone-based system
}

Jenny Drott, Victoria Fomichov Casaballe, Sussanne Börjeson and Carina Berterö

The self-archived postprint version of this journal article is available at Linköping University Institutional Repository (DiVA):

http://urn.kb.se/resolve?urn=urn:nbn:se:liu:diva-163675

N.B.: When citing this work, cite the original publication.

Drott, J., Fomichov Casaballe, V., Börjeson, S., Berterö, C., (2020), Sense of coherence and healthrelated quality of life in patients with neurotoxicity after cancer chemotherapy: Assessment from a real-time mobile phone-based system, Psycho-Oncology, 29(1), 107-113.

https://doi.org/10.1002/pon.5243

Original publication available at:

https://doi.org/10.1002/pon.5243

Copyright: Wiley (12 months)

http://eu.wiley.com/WileyCDA/ 
Sense of coherence and health-related quality of life in patients with neurotoxicity after cancer chemotherapy: assessment from a real-time mobile phone-based system

Short title: Mobile phone assessment of sense of coherence and quality of life

Jenny Drott, Victoria Fomichov, Sussanne Börjeson, Carina Berterö

Department of Surgery (Drott) and Department of Oncology (Börjeson), Division of Nursing

Science, Department of Medical and Health Sciences, Linköping University, Linköping, Sweden

(Drott, Börjeson and Berterö); and Centre for Organisational Support and Development County

Council of Östergötland, Linköping University, Linköping, Sweden (Fomichov)

\section{Corresponding author}

Dr Jenny Drott, Division of Nursing, Department of Medical and Health Sciences Linköping University, 58185 Linköping, Sweden

Email: jenny.drott@liu.se 


\begin{abstract}
Objective: In the present study, we aimed to assess sense of coherence (SOC) and health-related quality of life (HRQOL) during and after adjuvant chemotherapy by mobile phone-based reporting in patients with colorectal cancer experiencing neurotoxicity.

Methods: In this prospective descriptive cohort study a mobile phone-based system was used to receive a series of real time longitudinal patient-reported assessments of SOC (13-item), HRQOL (FACT-G 27-item) and neurotoxicity (OANQ 29-item) from 43 patients with colorectal cancer after being treated with chemotherapy including oxaliplatin. Measurements were conducted during the whole treatment period (mean 5 cycles) and up to 12 months after completing chemotherapy.

Results: In total, 817 questionnaire responses (226 SOC, 221 FACT-G, 370 OANQ) answered during and after chemotherapy treatment were available for analysis. Even though all patients experienced neurotoxicity during the treatment period, HRQOL was stable over time. Over time, the ratings of physical wellbeing tended to increase, while the subscale of social wellbeing tended to decrease. Overall SOC, including the three components comprehensibility, manageability and meaningfulness was stable during the entire study period. No internal data was missing due to the mobile phone-based system.
\end{abstract}

Conclusions: All patients had neurotoxicity during the treatment period that seemed to affect the social wellbeing component of HRQOL, but SOC seemed unaffected. Real-time patient-reported assessment using mobile phone technology could be valuable in the clinical setting to provide continuous individualised monitoring to help identify patients who need further evaluation to maintain or improve their psychosocial health. 


\section{Clinical trial registration}

ClinicalTrials.gov: NCT02412683.

Keywords: Cancer, Oncology, Cancer care, Chemotherapy, Colorectal cancer, Longitudinal study, mHealth, Neurotoxicity, Patient-reported outcomes 


\section{Background}

The platinum compound oxaliplatin (OXA) is a treatment option for patients who had surgery for colorectal cancer (CRC) and the first line treatment for those in a high risk of relapse. OXA is also a treatment option for patients in a palliative setting. OXA has been shown to improve survival, but treatment-related neurotoxicity is common. ${ }^{1-3}$ Research has shown that neurotoxicity may influence both physical and psychological distress, ${ }^{2-6}$ such as depression and sleep disturbance ${ }^{6,7}$ The impact of OXA-induced neurotoxicity on health-related quality of life (HRQOL) has been explored earlier in cross-sectional studies. ${ }^{8,9}$ Previous studies from our research team showed that patients balanced the neurotoxic side effects against their survival, striving to return to their former lives, i.e. before the diagnosis. Different strategies were used to manage the neurotoxicity and patients re-prioritised or avoided activities to be able to carry out daily activities. ${ }^{5,10}$

In one of our longitudinal studies, we showed that the high prevalence of OXAneurotoxic side effects significantly interfered with the patient's daily activities in different ways, both in the short and long term. The results highlight a need for tailored care for these patients. ${ }^{11}$ All patients had neurotoxicity that influenced activities in daily life, and therefore, it is important to investigate psychosocial and health issues deeper in this study.

Despite the effect of neurotoxicity on HRQOL and patient's daily life, little attention in earlier research has been paid to psychosocial aspects. Sense of coherence (SOC), developed by Antonovsky, ${ }^{12}$ has been used to explain psychosocial health during exposure to different types of stressors (e.g., cancer diagnosis). SOC is defined as the degree to which stressors are experienced as comprehensible, manageable and meaningful. Meaningfulness, the component that illustrates motivation, is seen as the most important. ${ }^{12}$ If a person thinks there is no reason to deal with 
challenges and no sense of meaning, then they will not be motivated to manage hard situations. Successful handling depends on the overall SOC, including its three components. Antonovsky ${ }^{12}$ divided overall SOC from high to low level, which gives eight possible types of sense of coherence levels. A high sense of coherence is a predictor of positive health outcomes. ${ }^{13,14}$ Patients with a high SOC may better understand their cancer treatment options, manage the aspects of their illness, and find a deeper meaning in their experience than those with a lower SOC. ${ }^{15,16}$

Monitoring side effects with mobile-phone technology (mHealth) have the potential to improve communication between patients and healthcare professionals. ${ }^{17} \mathrm{mHealth}$ opportunities can provide more effective care of symptoms, improve patient's health and quality of life.

mHealth can lead to a quicker response from professionals and may lead to lower inconvenience for patients. ${ }^{17}$ New possibilities to monitor side effects with mobile phone technology may be the future in the cancer care to improve patient health. Personality variables are associated with CRC patients' HRQOL independent of psychological distress and disease severity. ${ }^{18}$ In the present study, we aimed to assess mobile phone-based reporting of SOC and HRQOL during and after adjuvant chemotherapy reporting in patients with colorectal cancer experiencing neurotoxicity.

\section{Methods}

\section{Design}

The study has a prospective descriptive study design. 


\section{Participants}

Patients were recruited in four hospitals in Sweden from September 2013 to June 2016. Inclusion criteria for this study were as follows: patients with stage II-III CRC who were older than 18 years, could speak and understand Swedish and were receiving adjuvant OXA-based treatment. Exclusion criteria were patients with reduced cognitive function or earlier recognised neurotoxic side effects. Physicians decided if patients were able to participate, and the patients were given oral and written information about the study. Written informed consent was obtained. Forty-six patients were included and answered a series of questionnaires longitudinally, some of which are reported in this study. The study was performed in accordance with the Declaration of Helsinki and was approved by the Regional Ethical Review Board (record no: 2012/301-31).

\section{Measures}

\section{Real-time mobile phone-based system assessment}

Measures were performed in real time, using a mobile phone-based system (eSanté AB, http:// www.esante.se). ${ }^{24}$ OXA-induced neurotoxicity was measured two days after each OXA, and SOC and FACT-G was measured between the OXA chemotherapy treatments (a mean of 5 OXA treatments). Follow-up assessments were performed at 3, 6, 9 and 12 months after the last concluding OXA treatment. The results from frequency, severity and impact on daily activities

of neurotoxicity are reported separately. ${ }^{11}$ A study procedure was established that included instructions for nurses in using the mobile phone-based system. The patients received oral and written information from the nurses regarding installation of the system on their mobile phones (it was a platform-independent system compatible with JAVA ME phones, iPhone, Android, iPad, and Windows Phones). Most patients used their own mobile phones. The patient-reported 
data were transferred to a secure database and answers were available in real-time; web-based graphs were generated and could be viewed after logging in to a web interface.

SOC

To measure sense of coherence (SOC), a self-administered short version including 13item questionnaires was used to assess how the patient perceived comprehensibility, manageability and meaningfulness in their life situation. The component comprehensibility consists of five items, whereas manageability and meaningfulness only consist of four items each. Example of questions; Does it happen that you have feelings that you would rather not feel? How often do you have a feeling that there is little or no point in what you do in your daily life? Do you have a feeling that you are being unfairly treated?

The patients were asked to grade on a 7-point scale how they usually feel rather than how they currently feel; a low score indicated a low $\mathrm{SOC}^{19,20}$ An internal consistency testing of this 13-item scale produced a Cronbach's alpha ranging between 0.82 to $0.95 .^{12}$

\section{FACT-G}

HRQOL was measured using the FACT-G (Functional Assessment of Cancer TherapyGeneral). This 27-item self-report questionnaire measures responses on a five-point scale ranging from 0 (not at all) to 4 (very much). FACT-G have a recall period of the past 7 days. Example of statements from the questionnaire; I am bothered by side effects of treatment (physical wellbeing), I get emotional support from my family (social well-being), I am satisfied with how I am coping with my illness (emotional well-being) and I am able to enjoy life (functional well-being). Responses are summed to create a total score and subscale scores for physical, social, emotional 
and functional wellbeing, where higher scores reflect better HRQOL. ${ }^{21}$ The internal consistency testing of the subscales of the FACT-G was performed using Cronbach's alpha with an acceptable coefficient exceeding $0.70 .{ }^{22}$

\section{$O A N Q$}

To measure the frequency, severity and impact on daily activities of OXA-neurotoxic side effects, a 29-item questionnaire OANQ (Oxaliplatin-Associated Neurotoxicity Questionnaire) was used. The questionnaire is divided into three sections corresponding to the side effect locations: upper extremities, lower extremities and the face/mouth area. Patients first assessed whether they had the specific side effect, yes or no. ${ }^{11,23}$

Example; Do you have tingling? If they answered yes, they graded the side effects on a fivepoint scale ranging from $1=$ "hardly any" to $5=$ "very much". After that, they graded the impact of OXA-NTS on daily activities, $1=$ "hardly at all bothered" to $5=$ "extremely bothered" in a functional sense. ${ }^{11,23}$

\section{Data management and statistics}

Descriptive statistics for the clinical patient characteristics at baseline are expressed as the means and standard deviations (SDs), medians and ranges, or proportions. Longitudinal grading of SOC by domains and FACT-G separated by subscales were presented as dot charts to describe the evolution of the means (not adjusted) for each measure at each time points. For the purpose of this longitudinal design with unbalanced data, two linear mixed-effect models for repeated measures were adequate fitted with a first-order autoregressive covariance matrix. The SOC and the FACT-G are presented as the means with a $95 \% \mathrm{CI}$ adjusted for covariates, sex, age, OXA 
dose and neurotoxicity index as fixed effects and the patients as random effects. The neurotoxicity index was calculated by combining the grades of side effect severity and their effect on daily activities. The OXA dose entered was the average delivered dose until each specific time point and an interaction term (age*OXA dose) was added to the model due to the correlation between these two variables. The Akaike information criterion (AIC) was used to select the final models, and the final models considered an adequate fit. With a dichotomisation of the grading of side effects, the presence or not of neurotoxicity was analysed for each item. Since there was a need for a continuous and summarised measurement to perform aggregated longitudinal analysis, a Neurotoxicity Index was calculated by combining the grades for severity of side effects and their effect on daily activities. The following formula was developed for this purpose:

$$
\sum_{\text {item } 1}^{\text {item } 29}(\text { side effect grade } *(\text { effect on daily activities grade }+1))
$$

A total of maximum score in side effects (5= "very much") and impact on daily activities (5= “extremely bothered") generated a score of 30 in every item. The Neurotoxicity Index has the potential to measure from 0 up to the highest score of 870 . For the purpose of this longitudinal design with unbalanced data a linear mixed effect model for repeated measures with a first-order autoregressive covariance matrix was fitted. The Neurotoxicity Index is presented as mean with a $95 \%$ CI adjusted for covariates, sex, age and OXA dose as fixed effects and the patients as random effects. The OXA dose entered was the average delivered dose until each specific time point and an interaction term (age*OXA dose) was added to the model due to the correlation between these two variables. ${ }^{11}$

Due to the small sample size in the time points Cycle 7, the day before (d-1) to Cycle 11, and two days (d2) after OXA treatment, the values from these time points were excluded in all 
figures. For the purpose of this longitudinal study design with repeated assessments, graphs illustrate the patient-reported outcomes of neurotoxicity index and HRQOL and SOC over time. All tests had a significance level of $p<0.05$. All analyses were performed in IBM SPSS Statistics 25.

\section{Results}

\section{Characteristics of the sample}

Of the original 46 patients included in the main study assessing neurotoxicity, ${ }^{11} 43$ patients provided data for the present study (Table 1). In total, 817 questionnaire responses (226 SOC, 221 FACT-G and 370 OANQ) were available for analysis both during and postchemotherapy treatment. No internal data was missing due to the mobile phone-based system.

\section{HRQOL and SOC over time}

Even though all patients had neurotoxicity during the treatment period, all subscales in FACT-G were stable. The measures of FACT-G were increasing approximately from the treatment period start to the end of the follow-up. Despite the tendency to increase, there were no statistically significant differences between the follow-up assessments. The mean of the ratings in the subscale social wellbeing decreased after treatment and in the follow-up period, although not statistically significant.

The subscale physical wellbeing tended to increase over time, whereas the subscale of social wellbeing tended to decrease over time. The results show some improvement over the study period of all subscales but not in terms of the patient's social wellbeing (Figure 1). 
SOC, including all three components, was stable, even though the individual components decreased and increased synchronically during treatment. During the follow-up, meaningfulness did not change at all, whereas comprehensibility and manageability increased (Figure 2).

The mean of SOC and the components comprehensibility, manageability and meaningfulness during treatment and at 3, 6, 9- and 12-month follow-ups are shown in Figure 3.

The value was higher for comprehensibility than for the other components. Both Fact-G and SOC decreased and increased synchronically during treatment, and during follow-up, they showed a tendency to increase. Figure 3 shows the neurotoxicity index throughout the study period.

\section{Discussion}

In the current study, we explored patient reported SOC and HRQOL during and after chemotherapy in patients with CRC. The results showed that ratings of both HRQOL and SOC decreased and increased synchronically during treatment, and during follow-up, they showed a tendency to increase. The patient's physical wellbeing tended to increase over time, while the patient's social wellbeing tended to decrease over time. Our results suggest that social wellbeing may be affected due to neurotoxicity and problems adapting to daily activities after cancer diagnosis and treatment. Previous studies showed that patients learned to live with chronic neurotoxicity, ${ }^{3-5,11}$ and after a while, they normalised to the affected situation. ${ }^{5-8}$ The decline of social wellbeing, as found in this data set, measured mobile phone-based, confirms the quantitative research data.

A meta-ethnography approach review by Hildebrandt et al. ${ }^{25}$ synthesised patients with CRC experiences during treatment. According to their results, treatment impacts patients' daily 
life emotionally and physically; after completing chemotherapy, they still rely on others to help manage life. Gradually over time, the patients start learning to live with CRC and adapt to all the distress to return to a normal life. These results seem to be like our results regarding SOC and HRQOL. Patients with CRC tried to make sense of their experiences, find ways to manage their situation and find a meaning for their struggle. ${ }^{25}$ This is in agreement with our results that both SOC and FACT-G synchronically decreased and increased, respectively, during treatment, whereas during follow-up, they tended to increase.

At baseline, the HRQOL was like normative reference values. ${ }^{26}$ Notably, the mean scores of patients with colorectal cancer are similar to the mean scores for patients with other cancers, with minimally important difference scores of 5-7 for FACT-G.

It is noteworthy that the patients reported better HRQOL and the subscale physical wellbeing tended to increase over time, approximately from the treatment period start to the end of the follow-up. It could be that these patients experienced a true and notable improvement in their domain-specific HRQOL, but they may have also adapted to their new life situation, creating a "new normal," as mentioned in an interview study..$^{5}$ The patients' own statements about neurotoxicity and how the side effects affected their daily life showed that patients had developed different strategies to manage the neurotoxic side effects and re-prioritised or avoided activities to cope with their daily life. The patients expected to be cured by the cancer treatment, but instead, they realised they had to adapt to the remaining neurotoxic side effects and learn how to live with the side effects in daily life. ${ }^{5}$

Our results show that social wellbeing might have been affected due to neurotoxicity in the long term, and the side effects may influence psychosocial wellbeing. This may be due to patients struggling to cope with their condition, which consumes much of their energy, causing 
them to shy away from social activities. Although they adapt and learn to live with the problems in daily life, neurotoxicity can cause irritation, anger, and fatigue. In a previous study of Röhrl et al. ${ }^{27}$, the results show that lack of energy was the most occurring symptom post-chemotherapy in patients with CRC.

A lack of energy and depressive symptoms are common in patients diagnosed with cancer, and cancer patients with depression incur significantly higher visits and contacts with health care. ${ }^{28}$ Patients with neurotoxicity symptoms need careful attention from the health care team one year or more years after treatments to eliminate mis-estimation. ${ }^{29}$ Including and reporting patients views with a broad clinical perspective can truly reflect the risk and benefit balance. ${ }^{30}$ Patients with a strong SOC may protect against distress, anxiety and also depression. SOC may be useful for identifying patients who may be helped by psychological interventions. ${ }^{14}$ Counseling and stress management could then be considered. In a previous study of Paika et al. ${ }^{18}$ the results show that psychological distress and personality was associated with HRQOL in patients with CRC and independent of disease stage/severity. Studies showed a great approval for mHealth and mobile phone solutions in the oncology care among patients and health care professionals. ${ }^{17,31,32}$ The continuous monitoring of health-related quality of life via mobile systems can facilitate communication with the health care provider, leading to individualised support during treatment and enhanced social wellbeing in the long term. 


\section{Conclusions}

In conclusion, although all patients experienced neurotoxicity during treatment, the SOC was stable over time during and after treatment. However, the social wellbeing component of HRQOL decreased after treatment concluded. Both FACT-G and SOC showed synchronous variations during treatment, and during follow-up, they tended to increase, except for social wellbeing.

\section{Study limitations}

The strengths of the present study were the large number of assessments gathered in real time with mobile phone technology. The different measures and multidimensional ePROs completed at several time points during and after treatment gave a broad picture of patient wellbeing. However, there may be a risk that patients who felt more positively about the mobile phone system participated to a greater extent. This is a vulnerability in the study due to the small total sample size. A larger sample size may have provided more robust results. The small sample size in this study gave no statistical significance, which may limit the generalisability. However, this longitudinal study defines the trajectory of SOC and HRQOL over time in patients with neurotoxicity.

\section{Clinical implications}

Real-time patient-reported assessments can be valuable in the clinical setting to provide continuous individualised monitoring to help identify patients who need further evaluation or healthcare intervention to improve their psychosocial health. Mobile phone-based systems may facilitate communication and shared-decision making between patients and health care providers, which may enhance patient HRQOL. 


\section{Acknowledgements}

We would like to thank the patients who participated in the study and completed all the questionnaires over time.

\section{Conflict of interest statement}

The authors declare no conflict of interest.

\section{Ethical approval}

The patients were given oral and written information about the study and written informed consent was obtained. The study was performed in accordance with the Declaration of Helsinki and was approved by the Regional Ethical Review Board (record no: 2012/301-31).

\section{Data Availability Statement}

The data that support the findings of this study are available from the corresponding author upon reasonable request. 


\section{References}

1. Beijers AJ, Mols F, Tjan-Heijnen VC, Faber CG, van de Poll-Franse LV, Vreugdenhil G. Peripheral neuropathy in colorectal cancer survivors: the influence of oxaliplatin administration. Results from the population-based PROFILES registry. Acta Oncol 2015; 54:463-469.

2. Beijers AJ, Mols F, Vreugdenhil G. A systematic review on chronic oxaliplatin-induced peripheral neuropathy and the relation with oxaliplatin administration. Support Care Cancer 2014; 22:1999-2007.

3. Seretny M, Currie GL, Sena ES, Ramnarine S, Grant R, MacLeod MR, et al. Incidence, prevalence, and predictors of chemotherapy-induced peripheral neuropathy: a systematic review and meta-analysis. Pain 2014; 155:2461-2470.

4. Bennett BK, Park SB, Lin CS, Friedlander ML, Kiernan MC, Goldstein D. Impact of oxaliplatin-induced neuropathy: a patient perspective. Support Care Cancer 2012; 20:2959-2967.

5. Drott J, Starkhammar H, Kjellgren K, Bertero C. The trajectory of neurotoxic side effects' impact on daily life: a qualitative study. Support Care Cancer 2016; 24:3455-3461.

6. Tofthagen C. Surviving chemotherapy for colon cancer and living with the consequences. J Palliat Med 2010; 13:1389-1391.

7. Tofthagen C. Patient perceptions associated with chemotherapy-induced peripheral neuropathy. Clin J Oncol Nurs 2010; 14:E22-E28.

8. Tofthagen C, Donovan KA, Morgan MA, Shibata D, Yeh Y. Oxaliplatin-induced peripheral neuropathy's effects on health-related quality of life of colorectal cancer survivors. Support Care Cancer 2013; 21:3307-3313. 
9. Stefansson M, Nygren P. Oxaliplatin added to fluoropyrimidine for adjuvant treatment of colorectal cancer is associated with long-term impairment of peripheral nerve sensory function and quality of life. Acta Oncol 2016; 55:1227-1235.

10. Drott J, Starkhammar H, Kjellgren K, Bertero C. Neurotoxic side effects early in the oxaliplatin treatment period in patients with colorectal cancer. Oncol Nurs Forum 2018; 45:690-697.

11. Drott J, Fomichov V, Starkhammar H, Börjeson S, Kjellgren K, Berterö C. Oxaliplatininduced neurotoxic side effects and their impact on daily activities: a longitudinal study among patients with colorectal cancer. Cancer Nursing 2019. doi:

10.1097/ncc.0000000000000674.

12. Antonovsky A. The structure and properties of the sense of coherence scale. Soc Sci Med $1993 ; 36: 725-733$.

13. Pillay B, Lee SJ, Katona L, de Bono S, Burney S, Avery S. A prospective study of the relationship between sense of coherence, depression, anxiety, and quality of life of haematopoietic stem cell transplant patients over time. Psychooncology 2015; 24:220227.

14. Winger JG, Adams RN, Mosher CE. Relations of meaning in life and sense of coherence to distress in cancer patients: a meta-analysis. Psychooncology 2016; 25:2-10.

15. Antonovsky H, Sagy S. The development of a sense of coherence and its impact on responses to stress situations. J Soc Psychol 1986; 126:213-225.

16. Lindblad C, Langius-Eklof A, Petersson LM, Sackey H, Bottai M, Sandelin K. Sense of coherence is a predictor of survival: a prospective study in women treated for breast cancer. Psychooncology 2018; 27:1615-1621. 
17. Gurol-Urganci I, de Jongh T, Vodopivec-Jamsek V, Atun R, Car J. Mobile phone messaging reminders for attendance at healthcare appointments. Cochrane Database Syst Rev 2013:CD007458. doi: 10.1002/14651858.CD007458.pub3.

18. Paika V, Almyroudi A, Tomenson B, Creed F, Kampletsas EO, Siafaka V, et al. Personality variables are associated with colorectal cancer patients' quality of life independent of psychological distress and disease severity. Psychooncology 2010;19(3):273-82.

19. Antonovsky A, Elfstadius M. Hälsans mysterium (2. utg. / förord av Lennart Levi. ed., Natur och kulturs psykologiklassiker). Stockholm, Sweden: Natur och Kultur; 2005.

20. Antonovsky A. Unraveling the mystery of health: how people manage stress and stay well. San Francisco, CA: Jossey-Bass; 1987.

21. Cella DF, Tulsky DS, Gray G, Sarafian B, Linn E, Bonomi A, et al. The functional assessment of cancer therapy scale: development and validation of the general measure. $\mathrm{J}$ Clin Oncol 1993; 11:570-579.

22. Holzner B, Kemmler G, Cella D, de Paoli C, Meraner V, Kopp M, et al. Normative data for functional assessment of cancer therapy-general scale and its use for the interpretation of quality of life scores in cancer survivors. Acta Oncol 2004; 43:153-160.

23. Leonard GD, Wright MA, Quinn MG et al. Survey of oxaliplatin-associated neurotoxicity using an interview-based questionnaire in patients with metastatic colorectal cancer. BMC Cancer 2005; 16:5:116. 
24. Drott J, Vilhelmsson M, Kjellgren K, Bertero C. Experiences with a self-reported mobile phone-based system among patients with colorectal cancer: a qualitative study. JMIR Mhealth Uhealth 2016; 4:e66.

25. Hildebrandt C, Mayer H, Koller A. Experiences of patients with colorectal cancer from diagnosis until completion of treatment: a meta-ethnography approach. Psychooncology $2019 ; 28: 219-227$.

26. Cella D, Hahn EA, Dineen K. Meaningful change in cancer-specific quality of life scores: differences between improvement and worsening. Qual Life Res 2002; 11:207-221.

27. Rohrl K, Guren MG, Smastuen MC, Rustoen T. Symptoms during chemotherapy in colorectal cancer patients. Support Care Cancer 2019. doi: 10.1007/s00520-018-4598-y.

28. Mausbach BT, Yeung P, Bos T, Irwin SA. Health care costs of depression in patients diagnosed with cancer. Psychooncology 2018; 27:1735-1741.

29. Molassiotis A, Cheng HL, Lopez V, Au JSK, Chan A, Bandla A, et al. Are we misestimating chemotherapy-induced peripheral neuropathy? Analysis of assessment methodologies from a prospective, multinational, longitudinal cohort study of patients receiving neurotoxic chemotherapy. BMC Cancer 2019; 19:132.

30. Glimelius B, Nygren P. Lessons learned from the maturation of a cancer drug: oxaliplatin in colorectal cancer. Acta Oncol 2019; 58:395-397.

31. Kessel KA, Vogel MME, Schmidt-Graf F, Combs SE. Mobile apps in oncology: a survey on health care professionals' attitude toward telemedicine, mHealth, and oncological apps. J Med Internet Res 2016; 18:e312. 
32. Moradian S, Krzyzanowska MK, Maguire R, Morita PP, Kukreti V, Avery J, et al. Usability evaluation of a mobile phone-based system for remote monitoring and management of chemotherapy-related side effects in cancer patients: mixed-methods study. JMIR Cancer 2018; 4:e10932. 
Table 1. Patient and Clinical Characteristics

Age (years) mean (SD), median (range)

$\operatorname{Sex}^{1}$

Male

Social status

Living alone

Living with partner

Life partner and children at home,

$<21$ years

\section{Education level}

Less than high school

High school or college

University

Occupation

Type of cancer location, $\boldsymbol{n}(\%)$

Colon

Rectum

Chemotherapy, $n(\%)$

FOLFOX ${ }^{2}$

CAPOX $^{3}$

Oxaliplatin mg (total dose) mean, (range) ${ }^{4}$

1 Sex distribution in follow-up male/female;

3 months (55\%/45\%), 6 months $(50 \% / 50 \%)$,

9 months (40\%/60\%) and 12 months (46\%/54\%).

2 Fluorouracil + leucovorin + oxaliplatin

3 Capecitabine + oxaliplatin

4 Dose reduction was applied to all patients at some point, mean 5 OXA-treatments.
$62(8), 65(38-75)$

$n(\%)$

$27(63)$

5 (12)

$33(77)$

5 (12)

5 (12)

$23(54)$

14 (33)

$6(14)$

37 (86)

918, (120-1680) 


\section{Figure legends}

Figure 1. Health-related quality of life (FACT-G) during and after OXA-based chemotherapy. (PWB=Physical Wellbeing, SWB=Social Wellbeing, EWB=Emotional Wellbeing, $\mathrm{FWB}=$ Functional Wellbeing).

Figure 2. Sense of coherence (SOC) during and after OXA-based chemotherapy.

Figure 3. Estimated mean with mixed models of FACT-G and SOC in patients with OXAinduced neurotoxic side effects during and after treatment (adjusted for the covariates age, sex, OXA-dose/cycle). Neurotoxicity index (not the adjusted mean) presented over the entire study period. 


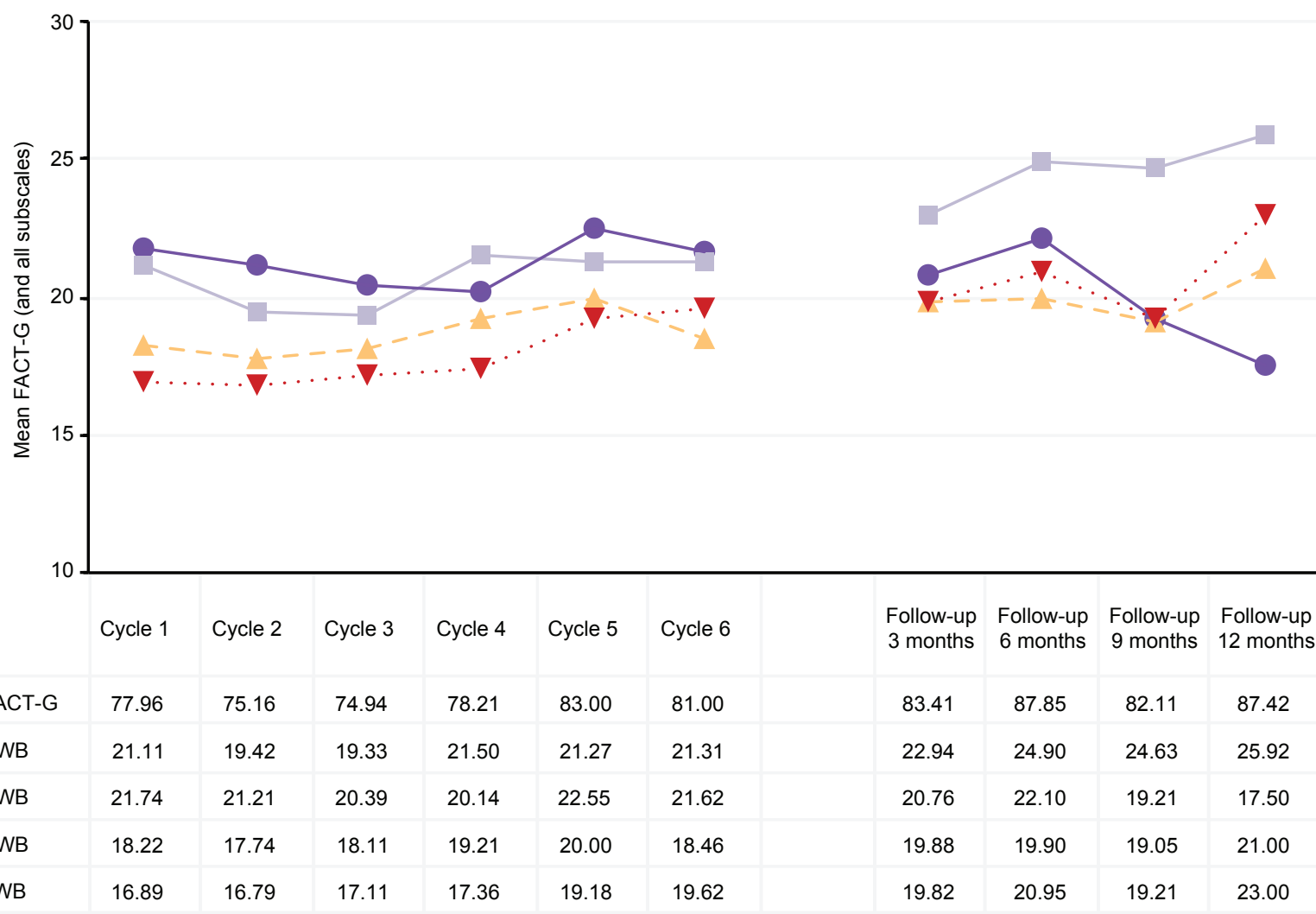




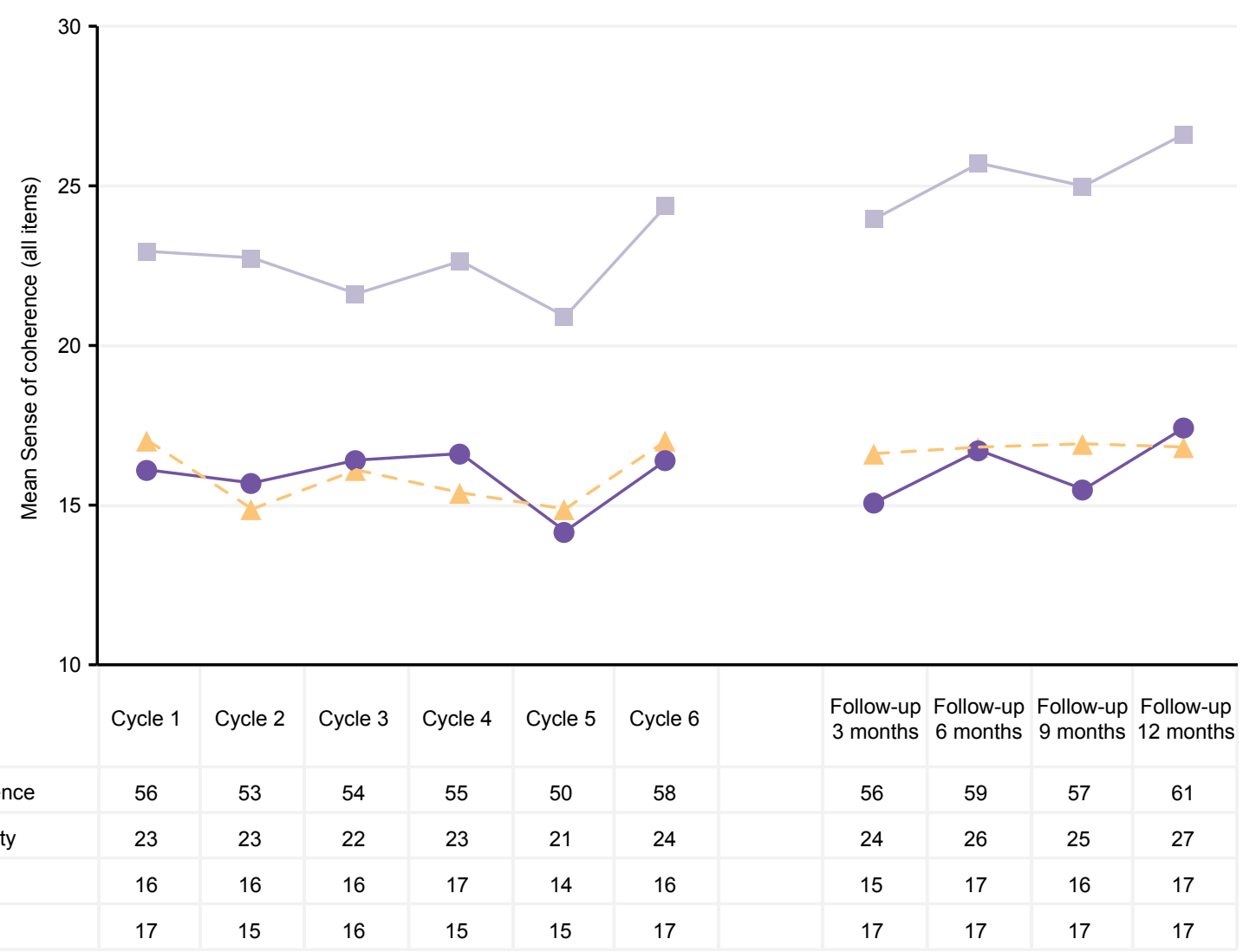




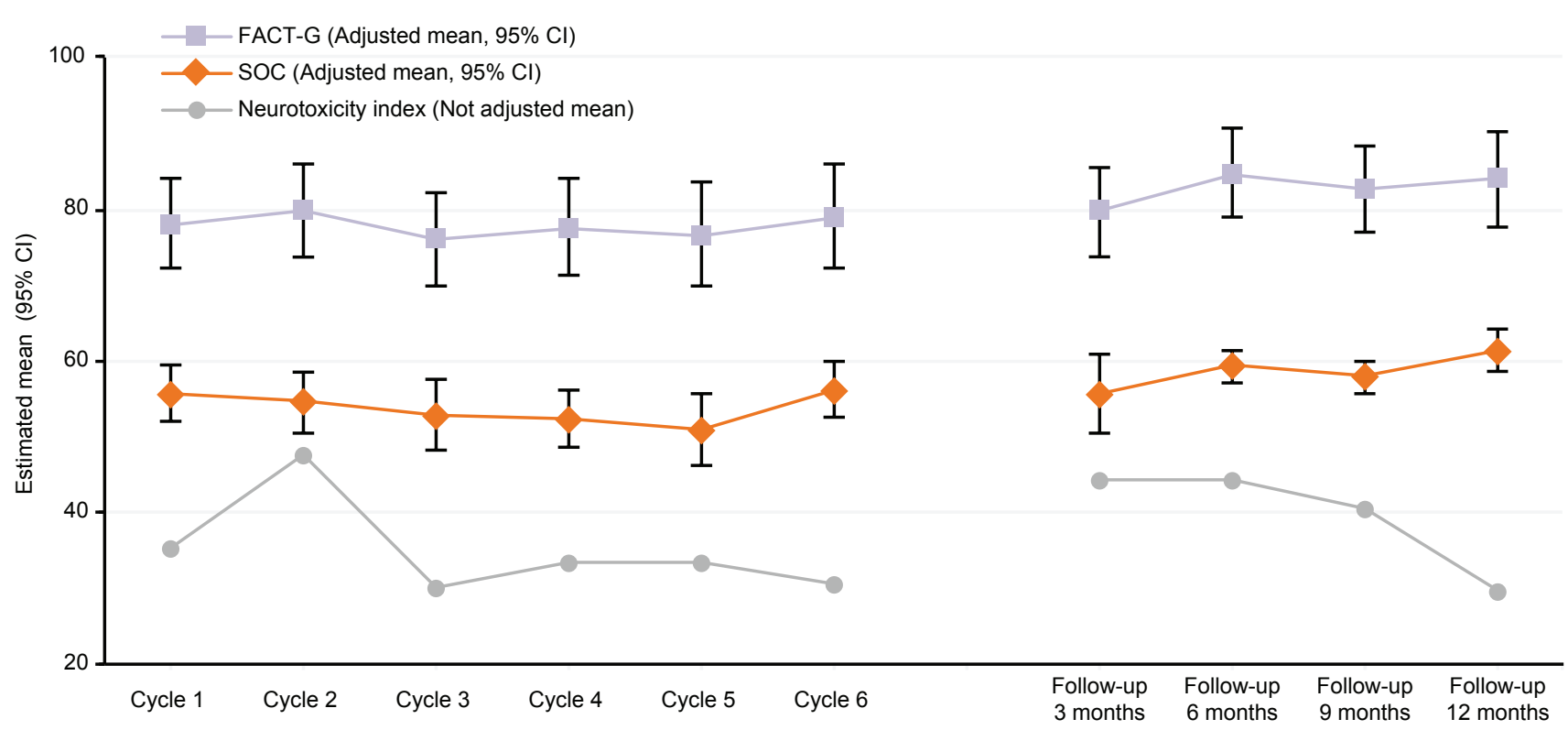

